\title{
PROPOSTA DE ALGORITMO DE CONDUTA CLÍNICA-FARMACÊUTICA PARA CASOS DE CONGESTÃo NASAL EM FARMÁCIA COMUNITÁRIA
}

Sarah Malta Monteiroํ, Fernanda de Cássia Freitas Barbosa² ${ }^{2}$ Lorrane Saliba da Silva ${ }^{3}$, Rhana Mariela Pinto Rodrigues ${ }^{1}$, Jessica Sampaio Batista ${ }^{5}$.

${ }^{1}$ Faculdade Estácio, ${ }^{2}$ Farmácia Pharmapele, ${ }^{3}$ Farmácia Bioflora, ${ }^{4}$ PSF Esmailda Marinho de Oliveira, ${ }^{5}$ Unidade de Pronto Atendimento de Castanhal

E-mail para correspondência: malta.sm@outlook.com

Submetido em: 25/05/2021 e aprovado em: 02/08/2021

\section{RESUMO}

Introdução: A congestão nasal, conhecida como "nariz entupido", é um sintoma resultante de estímulos nervosos que, por sua vez, provoca inflamação da mucosa nasal gerando, assim, a obstrução nasal. Diante do desconforto provocado pela congestão nasal, os indivíduos buscam aliviar este sintoma através de medicamentos de fácil acesso. Sendo assim, o farmacêutico é o responsável por manejar estes pacientes, assim como, orientá-los acerca do tratamento. Objetivos: Propor um algoritmo de conduta clínica-farmacêutica para casos de congestão nasal em farmácia comunitária. Metodologia: A partir de artigos indexados nas bases de dados eletrônicas e obedecendo os critérios de inclusão e exclusão, selecionaram-se artigos publicados no período entre 2010 e 2020 para a realização de uma revisão integrativa. Resultados e discussão: Através do levantamento bibliográfico e aplicação dos critérios de inclusão e exclusão nas buscas avançadas, apenas 63 artigos foram mantidos, dentre estes 36 foram removidos após leitura exploratória, restando apenas 27 artigos. De acordo com a pesquisa realizada, é de atribuição clínica do farmacêutico manejar pacientes com doenças autolimitadas. Diante disso, o farmacêutico pode fazer uso de mecanismos para ajudar na tomada de decisão universalizando, assim, a conduta do profissional desde o momento do acolhimento até a escolha do tratamento sendo este farmacológico ou não farmacológico. Conclusão: Infere-se que o desenvolvimento de algoritmo de decisão tende a uniformizar a conduta farmacêutica diante de um quadro clínico de congestão nasal, visando instruir os farmacêuticos, reduzir complicações causadas pela automedicação e promover saúde aos pacientes.

Palavras-chave: Algoritmo de manejo, Congestão Nasal, Descongestionantes Nasais, Orientação Farmacêutica.

\begin{abstract}
Introduction: Nasal congestion, known as "stuffy nose", is a symptom resulting from nervous stimuli which, in turn, causes inflammation of the nasal mucosa, thus generating nasal obstruction. Faced with the discomfort caused by nasal congestion, individuals seek to alleviate this symptom through easily accessible medications. Thus, the pharmacist is responsible for managing these patients, as well as guiding them about the treatment. Aims: To propose an algorithm for clinical-pharmaceutical management for cases of nasal congestion in a community pharmacy. Methodology: From articles indexed in electronic databases and obeying the inclusion and exclusion criteria, articles published in the period between 2010 and 2020 were selected for an integrative review. Results and discussion: Through the literature review and application of inclusion and exclusion criteria in advanced searches, only 63 articles were
\end{abstract}


kept, among these 36 were removed after exploratory reading, leaving only 27 articles. According to the research carried out, it is the pharmacist's clinical role to manage patients with self-limited diseases. Therefore, the pharmacist can use mechanisms to help in decision making, thus universalizing the professional's conduct from the moment of reception until the choice of treatment, whether pharmacological or non-pharmacological. Conclusion: It is inferred that the development of a decision algorithm tends to standardize pharmaceutical conduct in the face of a clinical picture of nasal congestion, aiming to instruct pharmacists, reduce complications caused by self-medication and promote health for patients.

Keywords: Management algorithm, Nasal congestion, Nasal Decongestants, Pharmaceutical Guidance.

\section{INTRODUÇÃO}

A congestão nasal, popularmente conhecida como "nariz entupido", é o sintoma clínico mais prevalente na população com rinite alérgica nos Estados Unidos, acometendo até 85\% dessa população ${ }^{(1)}$, Ademais, este sintoma gera no paciente dificuldade inalatória, desconforto, fadiga e até limitação das atividades diárias ${ }^{(2)}$. Além disso, pode ocorrer em todas as idades, estando associado a doenças crônicas (rinite alérgica), a doenças agudas (resfriado comum) e complicações de quadros gripais (sinusite/rinossinusite). Sendo assim, pode ser relacionado a causas estruturais, por exemplo adenoides, neoplasmas, desvio de septo nasal, hipertrofias das conchas nasais e pólipos nasais; há também correlação com fatores agravantes, a exemplo da mudanças na temperatura e alterações fisiológicas, como o estado gravídico ${ }^{(3)}$.

O nariz é o órgão externo da via respiratória, responsável por aquecer, filtrar e umidificar o ar inalado, permitindo o direcionamento deste ar e a proteção do trato respiratório inferior. Possui também função olfatória a qual permite a identificação de cheiros e odores do ambiente, a partir do contato do ar com a mucosa olfatória onde estão localizadas terminações do nervo olfatório que conduz o impulso nervoso até o bulbo olfatório no córtex cerebral, onde os odores são interpretados ${ }^{(4,5)}$.

Os cornetos fazem parte da estrutura nasal, objetivamente, são as paredes laterais da cavidade nasal, e possuem rica vascularização. Estes vasos, são controlados por fibras nervosas, cujo o papel é regular a permeabilidade vascular e de produzir muco ${ }^{(4)}$.

A congestão nasal, na maioria das vezes, ocorre devido a estímulos nervosos ativados por alérgenos ou antígenos capazes de gerar inflamação da mucosa nasal, ocorrendo vasodilatação local originando edema e, consequente, obstrução da via respiratória superior, espirros e $\operatorname{coriza}^{(4)}$.

A congestão nasal está presente, principalmente, em quadros gripais e de rinite alérgica, os indivíduos sintomáticos podem apresentar outros sintomas associados, sendo mais comum a perda do olfato e paladar, a dor nas "maçãs do rosto", o edema periorbitário, a secreção nasal, 
o gotejamento pós-nasal e a dor em supercílios. Das manifestações menos comuns, incluem-se tosse, dor ocular e dor em ouvidos ${ }^{(6,7)}$.

Estes indivíduos em busca de alívio do desconforto, buscam medicamentos de fácil acesso sem necessidade de prescrição médica. Dentre estes, destacam-se os descongestionantes nasais de uso tópico e anti-histamínicos ${ }^{(8)}$. Dá-se destaque ao primeiro que é altamente consumido, ao promover vasoconstrição local e alívio dos sintomas quase que imediato. Contudo, o uso indiscriminado deste, pode agravar o quadro congestivo, dado os efeitos colaterais de uso, como rinite medicamentosa, dependência psicológica e efeito rebote ${ }^{(9)}$.

Desta forma, entende-se a automedicação como o ato do paciente decidir, por conta própria, o medicamento que irá tomar. Esta pode ser motivada por acreditar que conhece a doença, não possuir recursos financeiros ou não possuir tempo disponível para busca de auxílio médico $^{(10,11,12)}$. Contudo, ao desconhecer o mecanismo de ação, posologia, interações medicamentosa e efeitos colaterais do medicamento escolhido, este pode ser utilizado de forma equivocada. Assim, ao invés de atingir a finalidade terapêutica, pode causar complicações e até mesmo o surgimento de novas doenças ${ }^{(13)}$.

Nesse contexto, o papel do farmacêutico, de acordo com a legislação vigente, é manejar pacientes acometidos com doenças autolimitadas, encaminhar este indivíduo para outro serviço de saúde caso apresente algum sinal de alerta, bem como orientar o uso racional de medicamentos que não necessitam de prescrição médica a fim de garantir as metas terapêuticas de cada fármaco. O presente trabalho é uma proposta de algoritmo de conduta clínicafarmacêutica para casos de congestão nasal em farmácia comunitária.

\section{MÉTODOS}

\section{Tipo de pesquisa}

Foi realizada revisão integrativa da literatura de caráter analítico-descritivo a partir de artigos indexados em bases de dados digitais a fim de desenvolver um algoritmo sobre o manejo clínico-farmacológico de pacientes com congestão nasal pelo profissional farmacêutico em âmbito de farmácia comunitária.

\section{Delineamento do estudo}

A proposta do algoritmo de conduta clínica-farmacêutica para casos de congestão nasal em farmácia comunitária contém dois algoritmos. O primeiro apresenta os critérios para decisão de encaminhamento considerando-se os aspectos como idade, sinais de alerta, outras patologias, 
situações fisiológicas $^{(2,3,14)}$, e o segundo algoritmo diz respeito ao manejo da congestão nasal na farmácia comunitária sendo elas as medidas farmacológicas e não farmacológicas como subsídio para tomada de decisão clínica ${ }^{(15)}$.

\section{Coleta de dados}

Foi realizada revisão de caráter analítico a partir de artigos indexados nas bases de dados: PUBMED, Google Acadêmico e SciElo. Os descritores foram determinados de acordo com os parâmetros de descritores MeSH, sendo a busca realizada com os descritores "congestão nasal", "descongestionante nasal", "anti-histamínico" e "solução salina”, os quais foram pesquisados separadamente e de forma articulada por meio de operador booleano "OR" em virtude do maior alcance.

\section{Critérios de inclusão e exclusão}

Dentre os artigos encontrados, foram aplicados critérios de inclusão norteadores para uma seleção mais criteriosa. Dentre estes, foram incluídos artigos publicados entre 2010 a 2020, disponíveis em língua portuguesa, inglesa e espanhola, os quais agregassem informações a respeito do manejo de congestão nasal pelo farmacêutico na farmácia comunitária. Dessa forma, foram considerados os artigos que obedeciam aos seguintes critérios de exclusão: artigos que contém relato de caso, comentários ou cartas, os artigos que apresentam resultados e discussões redundantes ou repetidos, não tratavam de mecanismo de ação e classe medicamentosa, como também apresentavam conteúdo superficial acerca da temática.

\section{Análise dos dados}

Inicialmente foram analisados títulos e resumos, visando conhecer o objetivo do artigo. Posteriormente, foi realizada leitura exploratória do manejo clínico farmacológico da congestão nasal e, por fim, redação do presente estudo. 
Figura 1. Fluxograma de seleção de artigos

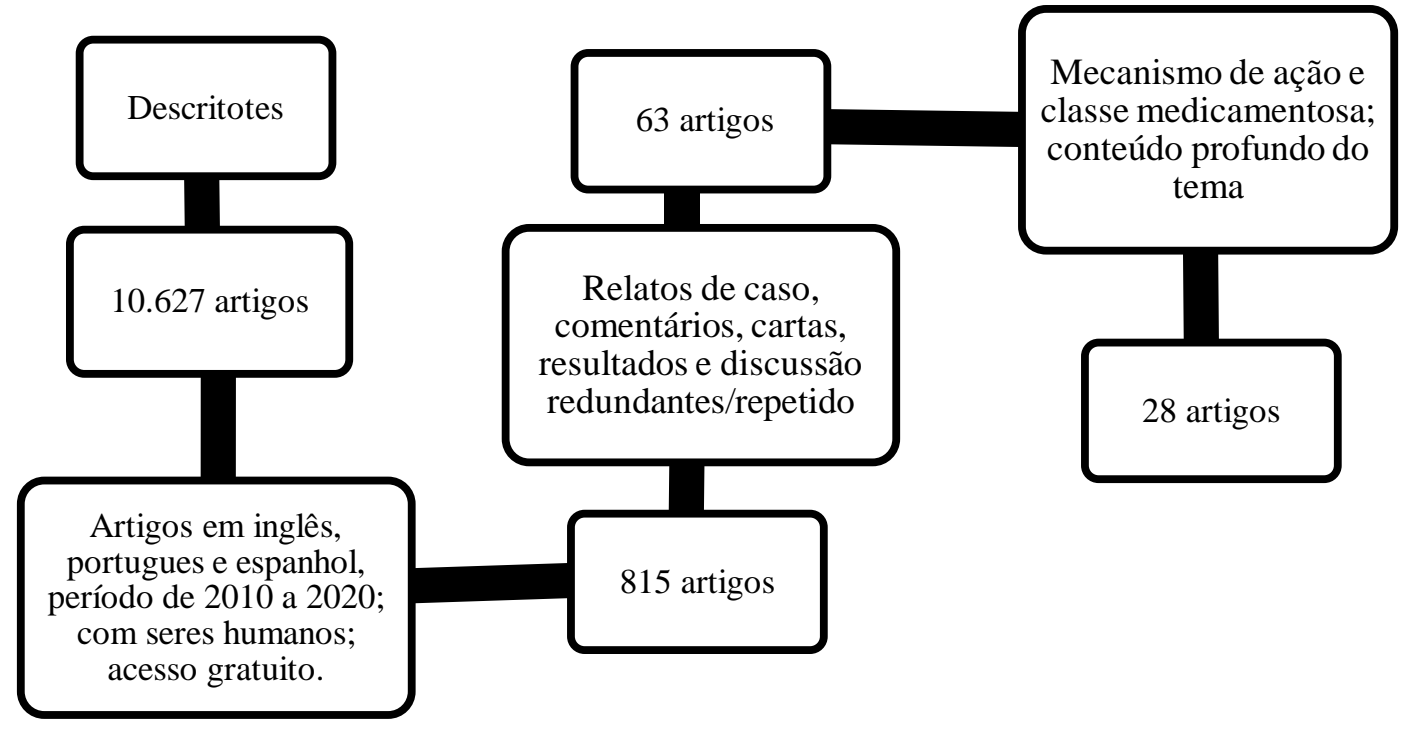

Fonte: Elaborado pelas autoras.

\section{RESULTADOS E DISCUSSÃO}

Foram selecionados 10.627 artigos por meio dos descritores nas bases de dados citadas, dos quais apenas 63 foram mantidos a partir da análise dos critérios de inclusão e exclusão, 36 foram removidos após leitura exploratória e seletiva por não relacionarem qualquer tipo de mecanismo de ação e classe medicamentosa, bem como terem abordado o tema proposto de forma superficial.

Figura 2. Base de dados coletada

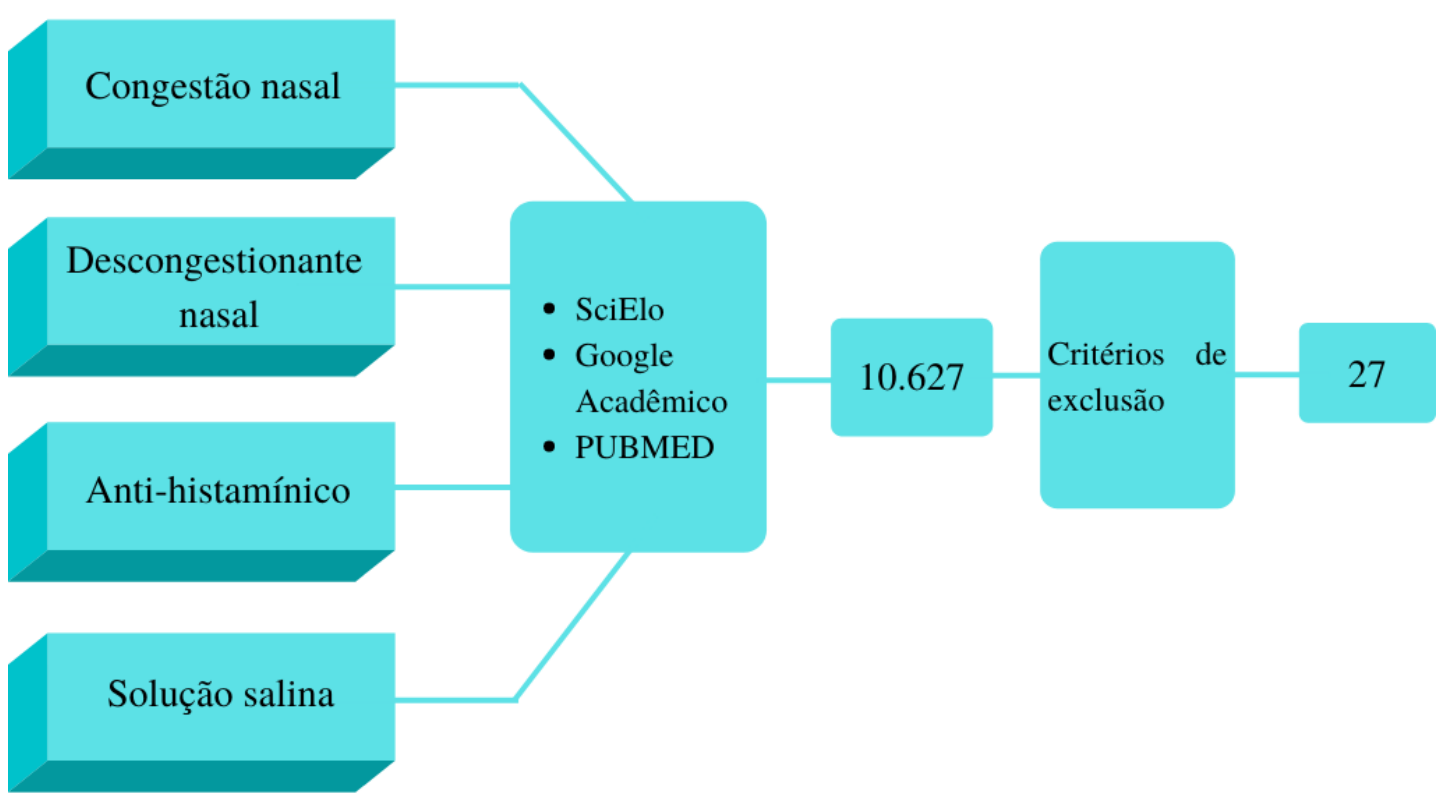

Fonte: Elaborado pelas autoras. 
Tabela 1. Artigos utilizados para a elaboração dos algoritmos

\begin{tabular}{cccc}
\hline Título & Autor(es) & Data de publicação & Local da publicação \\
\hline $\begin{array}{c}\text { Congestión nasal y rinorrea. } \\
\text { Problemas autolimitados } \\
\text { relacionados ao trato } \\
\text { respiratório. }\end{array}$ & Fried MP & 2020 & Manual Merck \\
Megier ET & 2017 & UNIJUI \\
$\begin{array}{c}\text { Clinical assessment, diagnosis, } \\
\text { and management of nasal } \\
\text { obstruction. }\end{array}$ & Esmaili A, & 2017 & Australian Family \\
$\begin{array}{c}\text { Prevalência da congestão } \\
\text { nasal em meio laboral }\end{array}$ & Gomes BF, & 2010 & Physician - AFP \\
hospitalar. & Cardoso SM, & & Rev Portug \\
& Arrobas A, Dias & & Otorrinolaringol e \\
& JG. & & \\
\hline
\end{tabular}

Fonte: Elaborado pelas autoras.

Dentre os 27 artigos mantidos, 14 abordavam principalmente sobre tratamento farmacológico entre outros assuntos, porém apenas 4 desses artigos tratavam sobre condições de inclusão e exclusão para o encaminhamento do paciente ao médico, portanto estas informações foram utilizadas para a produção dos algoritmos presentes nas Figuras 3 e 4.

\section{Atribuições clínicas do profissional farmacêutico}

As atribuições clínicas do farmacêutico foram regulamentadas na Resolução da Diretoria Colegiada (RDC) de $\mathrm{n}^{\circ} 585$ de 2013, a qual atribui ao profissional farmacêutico a responsabilidade de promover o uso racional de medicamentos, realizar anamnese farmacêutica, prover consulta farmacêutica, orientar o paciente, planejar e avaliar a farmacoterapia, identificar e avaliar interações ou incidentes relacionados a medicamentos, assim como, realizar intervenções farmacêuticas, elaborar o plano de cuidado, entre outros serviços, a fim de diminuir o índice de automedicação, visando a prevenção de doenças, proteção, recuperação e promoção à saúde. ${ }^{(16)}$

Também em 2013, foi regulamentada a RDC de no 586 que dispõe sobre a prescrição farmacêutica para o tratamento de doenças autolimitadas, como a congestão nasal originada de alergias, gripes e resfriados. ${ }^{(17)}$ Neste documento, ressalta-se a competência do farmacêutico, o qual é permitido prescrever somente Medicamentos Isentos de Prescrição (MIP) contidos na Lista de Grupos Terapêuticas Especificadas (GITE). Além disso, é consentido a indicação de 
acordo com a RDC $\mathrm{n}^{\mathrm{o}} 138$ de 2003, sobre o enquadramento na categoria de venda de medicamentos sem prescrição médica. ${ }^{(18,19)}$

\section{Conduta farmacêutica}

Dado as atribuições do farmacêutico, entende-se que este profissional está autorizado a acompanhar e manejar desordens autolimitadas, como a congestão nasal - sintoma secundário a infecções virais ou processos alérgicos. Para garantir a segurança na tomada de decisões, o uso de ferramentas adjuvantes acerca do manejo adequado, fazem-se necessárias. Dentre estas ferramentas, inclui-se protocolos validados, algoritmos de condução clínica e diretrizes terapêuticas baseados em evidências científicas. Assim, garante-se o acolhimento adequado do paciente, valorizando a presença de sinais de alerta e a necessidade de encaminhamento para outra área de cuidado da saúde, como representado na Figura 3. (2,3,14) $^{2}$

Estas ferramentas possibilitam a universalização da conduta do profissional quanto ao auxílio no acolhimento do paciente, na anamnese farmacêutica, à seleção do plano de cuidado individualizado e ao acompanhamento farmacoterapêutico, caso não haja o encaminhamento para outras áreas. ${ }^{(20)}$

Nesse contexto, o tratamento para congestão nasal pode ser farmacológico ou não farmacológico, como observado na Figura $4 .{ }^{(15)}$ Sendo uma possibilidade de tratamento o uso de MIP, apresentados na GITE. ${ }^{(19)}$

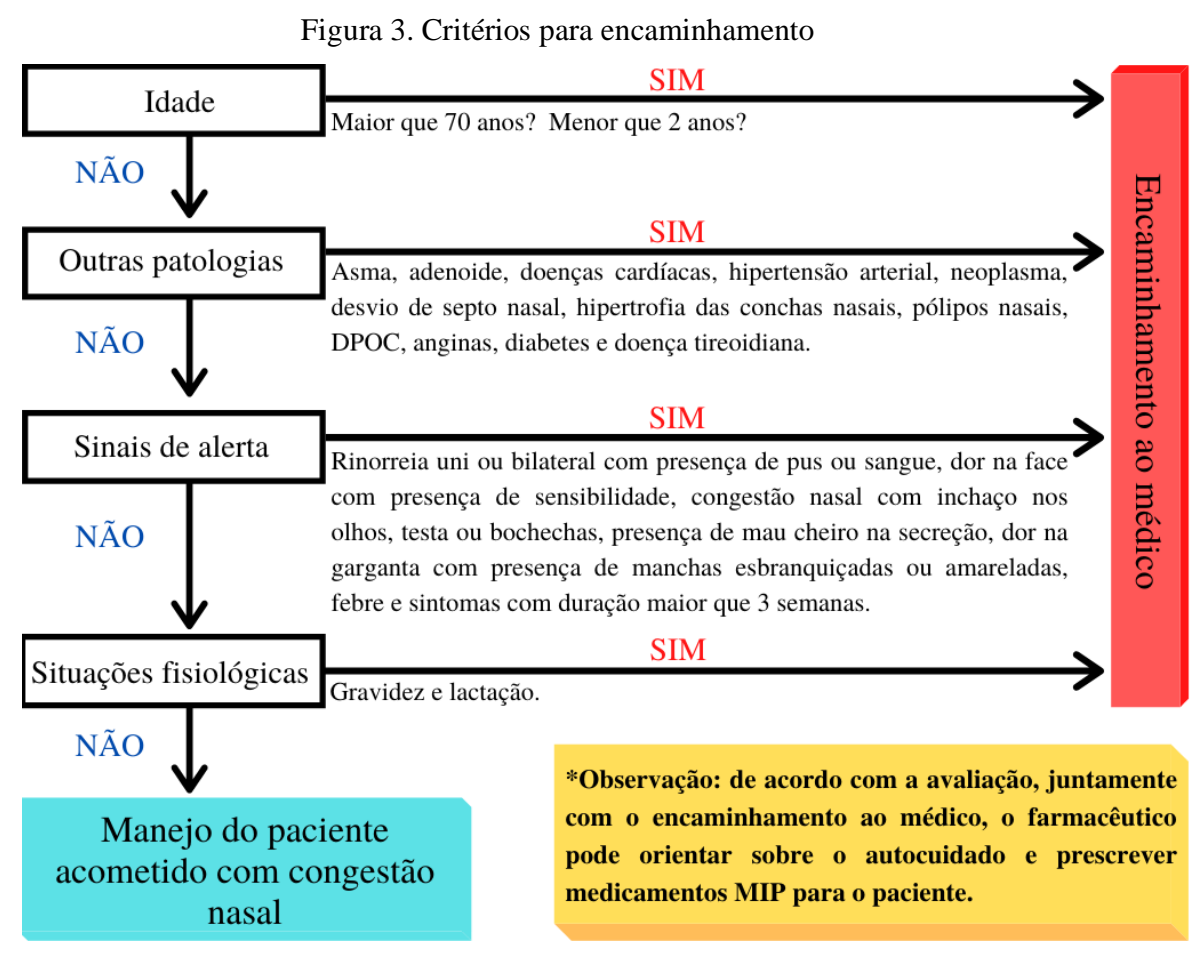

Fonte: Elaborado pelas autoras. 
Figura 4. Algoritmo de manejo para a congestão nasal

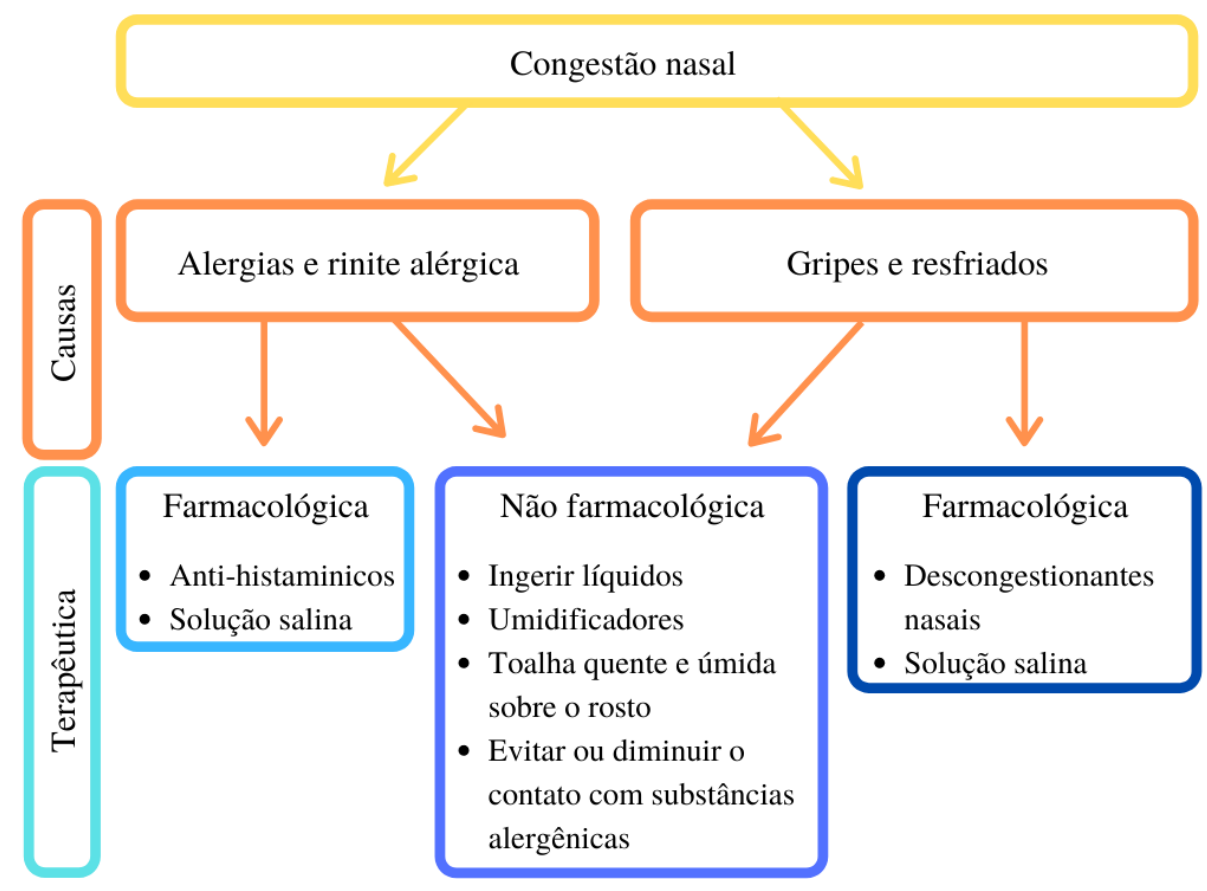

Fonte: Elaborado pelas autoras.

\section{Medicamentos recomendados}

\section{Descongestionantes nasais}

Dentre os medicamentos mais utilizados para aliviar a congestão nasal estão os descongestionantes nasais que são fármacos simpatomiméticas, as quais são responsáveis por promover a vasoconstrição local através da interação com os receptores adrenérgicos. ${ }^{(21)}$ Diminui o fluxo sanguíneo nasal, resultando na redução do edema e, posteriormente, proporcionando o alívio da congestão. ${ }^{(9,22)}$

Estes fármacos podem ser classificados em duas classes farmacológicas: (1) As aminas simpatomiméticas ou catecolaminas, como a fenilefrina, benzedrina e a pseudoefedrina. (2) Os derivados imidazólicos ou imidazolínicos, sendo seus representantes a clonidina, tetraidrozolina, oximetazolina, xilometazolina e a nafazolina. ${ }^{(23,24)}$

\section{Anti-histamínicos}

Outra classe farmacológica que é utilizada para tratamento da congestão nasal são os antihistamínicos. A histamina é mediadora de doenças alérgicas, dado que estimula as terminações nervosas sensoriais do nariz, provoca o aparecimento de espirros, prurido, aumento da permeabilidade vascular; gerando, assim, o edema e obstrução nasal, também estimula as 
secreções glandulares que, por sua vez, causam produção aumentada de muco, conhecido como rinorréia. ${ }^{(25,26)}$

Os anti-histamínicos controlam estes sintomas, visto que competem pelo receptor $\mathrm{H} 1 \mathrm{e}$ agem inibindo a ação da histamina, atuando na diminuição dos espirros e do prurido, assim como, a permeabilidade vascular e a rinorréia. ${ }^{(25)}$

\section{Solução Salina}

Para complementar o tratamento da congestão nasal, o farmacêutico pode prescrever as soluções salinas isotônicas $(0,9 \%)$ ou hipertônicas $(3 \%)$. Além de ser usada diariamente para a higiene nasal ou em condições de ressecamento nasal quando há uma baixa umidade relativa do ar, estas soluções também são responsáveis por provocar o amolecimento do muco, ajudar na diminuição dos agentes inflamatórios e, até mesmo, da inflamação. ${ }^{(27)}$

O Quadro 1, apresenta os medicamentos não tarjados, assim como a sua apresentação farmacêutica, posologia e modo de uso.

Quadro 1. Tratamento Farmacológico da congestão nasal (continua)

\begin{tabular}{|c|c|c|}
\hline Medicamento & Apresentação & Posologia e modo de uso \\
\hline Cloridrato de Fexofenadina & $\begin{array}{l}\text { Comprimidos revestidos contém } \\
60 \mathrm{mg}, 120 \mathrm{mg} \text { e } 180 \mathrm{mg} \text { de } \\
\text { Cloridrato de Fexofenadina. }\end{array}$ & $\begin{array}{l}01 \text { comprimido de } 60 \mathrm{mg} \text { duas vezes por dia; } \\
01 \text { comprimido de } 120 \mathrm{mg} \text {, uma vez por dia; } 01 \\
\text { comprimidos de } 180 \mathrm{mg} \text { uma vez por dia. }\end{array}$ \\
\hline Cloreto de Sódio 3\% & $\begin{array}{l}\text { Solução nasal contém 30mg/ml } \\
\text { de Cloreto de Sódio. }\end{array}$ & $\begin{array}{l}\text { Uma aplicação em cada narina, de } 6 / 6 \mathrm{~h} \text { ou até } \\
\text { de } 4 / 4 \mathrm{~h} \text {. }\end{array}$ \\
\hline Cloreto de Sódio 0,9\% & $\begin{array}{l}\text { Solução nasal contém } 9 \mathrm{mg} / \mathrm{ml} \text { de } \\
\text { Cloreto de Sódio. }\end{array}$ & $\begin{array}{l}\text { Aplicar a solução nas narinas conforme o } \\
\text { necessário. }\end{array}$ \\
\hline \multirow[t]{2}{*}{ Loratadina } & $\begin{array}{l}\text { Xarope contém } 1 \mathrm{mg} / \mathrm{ml} \text { de } \\
\text { Loratadina. }\end{array}$ & $\begin{array}{l}\text { Adultos e crianças acima de } 12 \text { anos: } 10 \mathrm{ml} \mathrm{de} \\
\text { loratadina, uma vez por dia. Não administrar } \\
\text { mais de } 10 \mathrm{ml} \text { em } 24 \text { horas; } \\
\text { Crianças de } 2 \text { a } 12 \text { anos ou peso corporal } \\
\text { abaixo de } 30 \mathrm{~kg} \text { : } 5 \mathrm{ml} \text { de loratadina, uma vez } \\
\text { por dia. Não administrar mais de } 5 \mathrm{ml} \text { em } 24 \\
\text { horas; } \\
\text { Peso corporal acima de } 30 \mathrm{~kg} \text { : } 10 \mathrm{ml} \text { de } \\
\text { loratadina, uma vez por dia. Não administrar } \\
\text { mais de } 10 \mathrm{ml} \text { em } 24 \text { horas. }\end{array}$ \\
\hline & $\begin{array}{l}\text { Comprimido contém } 10 \mathrm{mg} \text { de } \\
\text { Loratadina. }\end{array}$ & $\begin{array}{l}\text { Adultos e crianças acima de } 12 \text { anos ou com } \\
\text { peso corporal acima de } 30 \mathrm{~kg} \text { : } 01 \text { comprimido, } \\
\text { uma vez por dia. Não administrar mais de } 01 \\
\text { comprimido em } 24 \text { horas. }\end{array}$ \\
\hline \multirow{2}{*}{$\begin{array}{l}\text { Maleato de } \\
\text { Bronfeniramina associado } \\
\text { com Cloridrato de } \\
\text { Fenilefrina }\end{array}$} & $\begin{array}{l}\text { Comprimido contém } 12 \mathrm{mg} \text { de } \\
\text { Maleato de Bronfeniramina e } \\
15 \mathrm{mg} \text { de Cloridrato de } \\
\text { Fenilefrina. }\end{array}$ & $\begin{array}{l}\text { Adultos: } 01 \text { comprimido pela manhã e } 01 \\
\text { comprimido a noite. Dosagem máxima diária é } \\
\text { limitada a } 02 \text { comprimidos. }\end{array}$ \\
\hline & $\begin{array}{l}\text { Xarope a cada } 5 \mathrm{ml} \text { contém } 2 \mathrm{mg} \\
\text { de Maleato de Bronfeniramina e } \\
5 \mathrm{mg} \text { de Cloridrato de Fenilefrina. }\end{array}$ & $\begin{array}{l}\text { Adultos: de } 10 \text { a } 15 \mathrm{ml} \text {, de } 3 \text { a } 4 \text { vezes ao dia. } \\
\text { Dosagem máxima diária é limitada a } 60 \mathrm{ml} \text {. } \\
\text { Crianças acima de } 2 \text { anos: } 2,5 \text { a } 5 \mathrm{ml}, 4 \text { vezes } \\
\text { ao dia. Dosagem máxima diária é limitada a } \\
60 \mathrm{ml} \text {. }\end{array}$ \\
\hline
\end{tabular}


Quadro 1. Tratamento Farmacológico da congestão nasal (final)

\begin{tabular}{|c|c|c|}
\hline & $\begin{array}{l}\text { Solução oral (gotas) contém } 2 \mathrm{mg} \text { de } \\
\text { Maleato de Bronfeniramina e } 5 \mathrm{mg} \text { de } \\
\text { Cloridrato de Fenilefrina. }\end{array}$ & $\begin{array}{l}\text { Crianças acima de } 2 \text { anos: } 2 \text { gotas por kg } \\
\text { de peso dividida em } 3 \text { vezes ao dia, sendo } \\
\text { que } 1 \mathrm{ml} \text { equivale a } 20 \text { gotas. Dosagem } \\
\text { máxima diária é limitada a } 60 \text { gotas. }\end{array}$ \\
\hline \multirow{4}{*}{$\begin{array}{l}\text { Maleato de } \\
\text { Dexclorfeniramina }\end{array}$} & $\begin{array}{l}\text { Comprimidos contém } 2 \mathrm{mg} \text { de Maleato } \\
\text { de Dexclorfeniramina. }\end{array}$ & $\begin{array}{l}\text { Adultos e crianças acima de } 12 \text { anos: } 01 \\
\text { comprimido de } 3 \text { a } 4 \text { vezes por dia. Não } \\
\text { ultrapassar a dose máxima de } 06 \\
\text { comprimidos por dia. }\end{array}$ \\
\hline & $\begin{array}{l}\text { Drágeas repetabs contém } 6 \mathrm{mg} \text { de } \\
\text { Maleato de Dexclorfeniramina. }\end{array}$ & $\begin{array}{l}\text { Adultos e crianças acima de } 12 \text { anos: } 01 \\
\text { drágea pela manhã e outra ao deitar. }\end{array}$ \\
\hline & $\begin{array}{l}\text { Solução gotas contém } 0,4 \mathrm{mg} / \mathrm{ml} \text { de } \\
\text { Maleato de Dexclorfeniramina. }\end{array}$ & $\begin{array}{l}\text { Adultos e crianças acima de } 12 \text { anos: } 20 \\
\text { gotas de } 3 \text { a } 4 \text { vezes por dia. Não } \\
\text { ultrapassar a dose máxima de } 120 \text { gotas } \\
\text { por dia; } \\
\text { Crianças de } 6 \text { a } 12 \text { anos: } 10 \text { gotas ou } 1 \text { gota } \\
\text { a cada } 2 \mathrm{~kg} \text { de peso, } 3 \text { vezes por dia. Não } \\
\text { ultrapassar a dose máxima de } 60 \text { gotas por } \\
\text { dia; } \\
\text { Crianças de } 2 \text { a } 6 \text { anos: } 5 \text { gotas ou } 1 \text { gota a } \\
\text { casa } 2 \mathrm{~kg} \text { de peso, } 3 \text { vezes por dia. Não } \\
\text { ultrapassar a dose máxima de } 30 \text { gotas por } \\
\text { dia. }\end{array}$ \\
\hline & $\begin{array}{l}\text { Xarope contém } 0,4 \mathrm{mg} / \mathrm{ml} \text { de Maleato } \\
\text { de Dexclorfeniramina. }\end{array}$ & $\begin{array}{l}\text { Adultos e crianças acima de } 12 \text { anos: } 5 \mathrm{ml} \text {, } \\
\text { de } 3 \text { a } 4 \text { vezes por dia. Não ultrapassar a } \\
\text { dose máxima de } 30 \mathrm{ml} \text { por dia; } \\
\text { Crianças de } 6 \text { a } 12 \text { anos: } 2,5 \mathrm{ml}, 3 \text { vezes por } \\
\text { dia. Não ultrapassar a dose máxima de } \\
15 \mathrm{ml} \text { por dia; } \\
\text { Crianças de } 2 \text { a } 6 \text { anos: } 1,25 \mathrm{ml}, 3 \text { vezes por } \\
\text { dia. Não ultrapassar a dose máxima de } \\
7,5 \mathrm{ml} \text { por dia. }\end{array}$ \\
\hline \multirow{2}{*}{$\begin{array}{l}\text { Paracetamol associado } \\
\text { com Maleato de } \\
\text { Clorfeniramina e } \\
\text { Cloridrato de Fenilefrina }\end{array}$} & $\begin{array}{l}\text { Cápsulas contém 400mg de } \\
\text { Paracetamol, 4mg de Maleato de } \\
\text { Clorfeniramina e } 4 \mathrm{mg} \text { de Cloridrato de } \\
\text { Fenilefrina. }\end{array}$ & $\begin{array}{l}\text { Adultos de } 18 \text { a } 60 \text { anos: } 01 \text { cápsula a cada } \\
4 \text { horas. Não ultrapassar } 05 \text { cápsulas por } \\
\text { dia. }\end{array}$ \\
\hline & $\begin{array}{l}\text { Solução oral contém } 40 \mathrm{mg} / \mathrm{ml} \text { de } \\
\text { Paracetamol, } 0,6 \mathrm{mg} / \mathrm{ml} \text { de Maleato de } \\
\text { Clorfeniramina e } 0,6 \mathrm{mg} / \mathrm{ml} \text { de } \\
\text { Cloridrato de Fenilefrina. } \\
\end{array}$ & $\begin{array}{l}\text { Adultos de } 18 \text { a } 60 \text { anos: } 10 \mathrm{ml} \text { a cada } 4 \\
\text { horas. Não ultrapassar } 40 \mathrm{ml} \text { por dia. }\end{array}$ \\
\hline $\begin{array}{l}\text { Maleato de } \\
\text { Carbinoxamina } \\
\text { associado ao } \\
\text { Paracetamol e Cloridrato } \\
\text { de Fenilefrina }\end{array}$ & $\begin{array}{l}\text { Comprimido branco contém 400mg de } \\
\text { Paracetamol, comprimido amarelo } \\
\text { contém } 400 \mathrm{mg} \text { de Paracetamol e } 20 \mathrm{mg} \\
\text { de Cloridrato de Fenilefrina e o } \\
\text { comprimido laranja contém } 400 \mathrm{mg} \text { de } \\
\text { Paracetamol e } 4 \mathrm{mg} \text { de Maleato de } \\
\text { Carbinoxamina. }\end{array}$ & $\begin{array}{l}\text { Adultos e crianças acima de } 12 \text { anos: pela } \\
\text { manhã tomar } 02 \text { comprimidos } \\
\text { (comprimido } 01 \text { amarelo }+01 \text { branco); } \\
\text { Pela tarde, tomar } 02 \text { comprimidos }(01 \\
\text { amarelo + } 01 \text { branco); E pela noite tomar, } \\
02 \text { comprimidos ( } 01 \text { amarelo + } 01 \text { laranja). } \\
\text { Respeitando o intervalo de } 8 / 8 \text { horas entre } \\
\text { as doses. Não ultrapassar as } 3 \text { doses em } 24 \\
\text { horas. }\end{array}$ \\
\hline
\end{tabular}

Fonte: Adaptado do Bulário Eletrônico disponibilizados pela Vigilância Sanitária do Brasil. ${ }^{(28)}$ 


\section{CONCLUSÃO}

Para o tratamento de congestão nasal no âmbito permitido ao farmacêutico existem medidas farmacológicas ou não farmacológicas. A exemplo de manejo farmacológico de gripes e resfriados, indica-se descongestionantes nasais e solução salina; para causas alérgicas, utilizase solução salina associado a anti-histamínicos (Loratadina, Maleato de dexclorfeniramina) ou solução salina associado a anti-histamínicos compostos (Maleato de Bronfeniramina com Cloridrato de Fenilefrina; Paracetamol com Maleato de Carbinoxamina e Cloridrato de Fenilefrina). Dentre as medidas não farmacológicas, recomenda-se hidratação, repouso e redução de contato com alérgenos (para alergias).

Por fim, este estudo apresenta uma proposta de algoritmo de conduta clínicafarmacêutica para casos de congestão nasal em farmácias comunitárias, com finalidade de auxiliar no momento de tomada de decisão sobre o manejo clínico mais adequado para cada paciente. Vale salientar, que por este ser uma proposta de algoritmo, faz-se necessária validação do mesmo para uniformizar o atendimento.

\section{REFERÊNCIAS BIBLIOGÁFICAS}

1. Baraniuk JN. Subjective nasal fullness and objective congestion. Proc Am Thorac Soc. 2011 Mar;8(1):62-69. 10.1513/pats.201006-042RN.

2. Gomes BF, Cardoso SM, Arrobas A, Dias JG. Prevalência da congestão nasal em meio laboral hospitalar. Rev Portug Otorrinolaringol e Cirurg. Cérvico-Facial. 2010 Set;48(3):123130.https://doi.org/10.34631/sporl.222

3. Esmaili A, Acharya A. Clinical assessment, diagnosis and management of nasal obstruction. Aust Fam Physician. 2017 Jul;46(7):499-503.

4. Ferrito MJ. Resistência nasal. Lisboa. Dissertação [Mestrado Integrado em Medicina] Faculdade de Medicina da Universidade de Lisboa; 2016.

5. Associação Brasileira de Otorrinolaringologia e Cirurgia Cérvico-Facial- ABORL-CCF, Associação Brasileira de Alergia e Imunologia-ASBAI. III Consenso brasileiro sobre de rinites. Braz J Otorhinolaryngol. 2012 nov/dez;75(6):52p.

6. Basuari G. ¿Cómo puede ayudarnos la Homeopatía en el tratamiento de las sinusitis? In: Hablando de homeopatia [internet]. Cidade. 2019 [acesso em 25 set 2020]. Disponível em: https://www.hablandodehomeopatia.com/puede-ayudarnos-la-homeopatia-tratamiento-las-sinusitis/

7. Healthy Children. [internet]. Dolor o congestión de los senos paranasales (sinusitis). [acesso em 25 set 2020]. Disponível em: https://www.healthychildren.org/Spanish/tips-tools/symptomchecker/Paginas/symptomviewer.aspx?symptom=Dolor+o+Congesti\%C3\%B3n+de+Los+Sen os+Paranasales+(Sinusitis)

8. Lenz D, Cardoso KS, Bitti ACR, Andrade TU. Evaluation of the use of topical nasal descongestants in university students from health sciences courses. Braz J Pharm Sci. 2011 out/dez;47(4):761-767. https://doi.org/10.1590/S1984-82502011000400013 
9. Fernandes TRG. Automedicação e descongestionantes nasais: riscos de intoxicação. Brasília: Monografia [Graduação em Biomedicina] - Faculdade de Ciências da Educação e Saúde; 2018.

10. Montanari CM, Souza WA, Vilela DO, Araújo FS, Podestá MHMC, Ferreira EB. Automedicação em acadêmicos de uma universidade pública do sul de Minas Gerais., Tempus acta saúde colet. 2014 dez;8(4):257-268. https://doi.org/10.18569/tempus.v8i4.1596

11. Matos JF, Pena DAC, Parreira MP, Santos TC, Coura-Vital W. Prevalência, perfil e fatores associados a automedicação em adolescentes e servidores de uma escola pública profissionalizante. Cad Saúde Colet. 2018;26(1):76-83. Doi: 10.1590/1414462X201800010351

12. Gama ASM, Secoli SR. Automedicação em estudantes de enfermagem no estado do Amazonas - Brasil. Rev Gaúcha Enferm. 2017 mar;38(1):e65111. https://doi.org/10.1590/19831447.2017.01.65111

13. Pereira DTM, Neto ELV, Cruz NPS. Perfil da automedicação entre idosos assistidos por unidades básicas de saúde. Rev Enferm UFPE on line. 2014 nov;8(11):3868-3873. DOI: 10.5205/reuol.6679-58323-1-ED.0811201408

14. Fried MP. Congestión nasal y rinorrea. In: Manual merck - versão profissional [internet]. Disponível em: https://www.merckmanuals.com/es-pr/professional/trastornosotorrinolaringol\%C3\%B3gicos/abordaje-del-paciente-con-s\%C3\%ADntomas-nasales-yfar\%C3\%ADngeos/congesti\%C3\%B3n-nasal-y-rinorrea

15. Megier ET. Problemas autolimitados relacionados ao trato respiratório. Rio Grande do Sul. Monografia [Especialização em Farmácia Hospitalar] - Universidade Regional do Noroeste do Estado do Rio Grande do Sul; 2017.

16. Conselho federal de farmácia (Brasil). Resolução $\mathrm{n}^{\circ} 585$ de 29 de agosto de 2013. Regulamenta as atribuições clinicas do farmacêutico e dá outras providencias. Capitulo I artigo $7^{\circ}$. Diário oficial da união 25 set 2013;Seção 1.

17. Conselho federal de farmácia CFF. Resolução no 586 de 29 de Agosto de 2013. Regula a prescrição farmacêutica e dá outras prividências. Diário oficial da união 26 set 2013;Seção 1.

18. Agência Nacional de Vigilância Sanitária (Brasil). Resolução no 138 de 29 de maio de 2003. Produtos isentos de prescrição medica; Capitulo I artigo $1^{\circ}$. Diário Oficial da União 02 jun 2003, republicada em 06 jan 2004; Seção 1.

19. Agência Nacional de Vigilância Sanitária (Brasil). Resolução - $\mathrm{n}^{\circ} 138$, de 29 de maio de 2003. Produtos isentos de prescrição medica; capitulo II artigo $2^{\circ}$. Diário Oficial da União 02 jun 2003, republicada em 06 jan 2004; Seção 1.

20. Conselho federal de farmácia. PROFAR - Programa de Suporte ao Cuidado Farmacêutico na Atenção à Saúde. Guia de prática clínica sinais e sintomas respiratórios: espirro e congestão nasal. Brasília; 2016. [Internet]. Disponivel em: https://www.cff.org.br/userfiles/file/Profar_vol1_FINAL_TELA.pdf

21. Mello Jr JF, Mion OG, Andrade NA, Anselmo-Lima WT, Stamm AEC, Almeida WLC, et al. Brazilian academy of rhinology position paper on topical intranasal therapy. Braz $\mathbf{J}$ Otorhinolaryngol. 2013;79(3):391-400. DOI: 10.5935/1808-8694.20130067

22. Santos ARF. Descongestionantes nasais - perfil de utilização, efeitos adversos e interações medicamentosas. Covilhã. Dissertação [Mestrado em Ciências Farmacêuticas] - Universidade da Beira Interior; 2014. 
23. Souza JKC, Batista LM, Assis TS. Utilização de descongestionantes nasais por acadêmicos do curso de farmácia da Universidade Federal da Paraíba. In: Revista Interdisciplinar de Ciências Médicas- Anais. 2017;1(1).

24. Castro LN, Mello MM, Fernandes WS. Avaliação da prática de automedicação com descongestionantes nasais por estudantes da área da saúde. J Health Sci Inst. 2016 jul/set;34(3):163-167.

25. Rodrigues AIS. Anti-histamínicos em otorrinolaringologia. Lisboa. Dissertação [Mestrado Integrado em Medicina]- Faculdade de Medicina em Lisboa; 2018.

26. MedlinePlus[internet]. Rinorrea o congestión nasal en adultos [acesso em 25 set 2020]. Disponível em: https://medlineplus.gov/spanish/ency/article/003049.htm

27. Olbrich Neto J, Olbrich SRLR, Mori NLR, de Oliveira AE, Corrente JE. Variations in peak nasal inspiratory flow among healthy students after using saline solutions. Braz J Otorhinolaryngol. 2016;82(2):184-190. https://doi.org/10.1016/j.bjorl.2015.03.012.

28. Brasil, Ministério da Saúde. Bulário eletrônico da ANVISA [homepage na internet] [acesso em 25 set 2020]. Disponivel em https://consultas.anvisa.gov.br/\#/bulario/ 